\title{
Sentipensarnos como Universidad Pública, profundizar la Universidad Pública como derecho: dos caras de una misma moneda
}

\author{
Feel and think like a Public University, deepen the Public \\ University as a right: two sides of the same coin
}

\section{María Inés Peralta}

https://orcid.org/0000-0001-8637-4764

mariainesperalta50@gmail.com

Universidad Nacional de Córdoba | Argentina

\section{RESUMEN}

En primer lugar me interesa resaltar el contexto de producción de las ideas y prácticas desde las que aquí se escribe y que nos ubica en la Universidad Pública Argentina. Universidad que viene siendo profundamente atacada, por el ahogo presupuestario, por concepciones meritocráticas, por cooptaciones mercantilistas, por criterios universales (del poder del norte) negadores de las diferencias (del sur). Estos son los rasgos de una universidad pública debilitada que necesita el proyecto reaccionario que hoy despliega sus estrategias para instalarse en nuestra Latinoamérica y en el mundo.

Si hoy nosotros/as afirmamos que la educación superior es un derecho es porque hubo actores sociales y políticos que trabajaron, estudiaron, fundamentaron, confrontaron y se movilizaron por ello.

\section{ABSTRACT}

In the first place, I am interested in highlighting the context of production of ideas and practices from which it is written here and which places us in the Argentine Public University. University that has been deeply attacked, due to budgetary drowning, meritocratic conceptions, mercantile co-optations, universal criteria (of the power of the north) denying differences (of the south). These are the features of a weakened public university that needs the reactionary project that today deploys its strategies to settle in our Latin America and in the world.

If today we affirm that higher education is a right, it is because there were social and political actors who worked, studied, substantiated, confronted and mobilized for it.

PALABRAS CLAVE Universidad Pública, Derechos, Educación Superior.
KEY WORDS

Public University, Rights,

Higher Education. 
En primer lugar me interesa resaltar el contexto de producción de las ideas y prácticas desde las que aquí se escribe y que nos ubica en la Universidad Pública Argentina. Universidad que viene siendo profundamente atacada, por el ahogo presupuestario, por concepciones meritocráticas, por cooptaciones mercantilistas, por criterios universales (del poder del norte) negadores de las diferencias (del sur). Estos son los rasgos de una universidad pública debilitada que necesita el proyecto reaccionario que hoy despliega sus estrategias para instalarse en nuestra Latinoamérica y en el mundo.

Si hoy nosotros/as afirmamos que la educación superior es un derecho es porque hubo actores sociales y políticos que trabajaron, estudiaron, fundamentaron, confrontaron y se movilizaron por ello.

La gesta revolucionaria estudiantil de la Reforma del 18 instaló el cogobierno. Desde allí nuestra universidad pública es impensable sin la participación de todos los claustros -o colectivos- en la toma de decisiones, lo que nos abre al necesario debate entre distintas visiones de cada momento sobre las necesidades, las expectativas, las reivindicaciones, lo justo y lo injusto, de docentes, estudiantes, egresados y no docentes.

A fines de la década del 40 , se sancionó la gratuidad de la universidad pública argentina y con ello uno de los principales aspectos en el que se asienta la legitimidad de la universidad: sin la gratuidad, el acceso a la educación superior es una mezcla de mérito y privilegio que oculta las discriminaciones interseccionales de clase, raza, género o etnia.

En la década de los 60/70, en las que hubo incontables acontecimientos -de los cuales yo no puedo dejar de nombrar al Cordobazo- en los que la universidad se reconoció como actor político ineludible en la construcción de una sociedad movilizada por la libertad y la justicia, debatiendo el papel del conocimiento en esa construcción, con vocación latinoamericanista y con un efectivo y activo compromiso social.

La Universidad de la década del 80, de la recuperación democrática, comenzó a gestarse desde la necesidad silenciosa de desocultar lo oculto, de vencer el miedo, de recuperar memoria colectiva. Fue la Universidad que se movilizó masivamente ante cada nuevo intento golpista de parte de fuerzas armadas que insistían en reinstalar los momen- 
tos más negros de nuestra historia. Ante esto, estuvo entera, en la calle, movilizada por el Nunca Más.

La Universidad de los 90 fue la de las marchas multitudinarias por todo el país, resistiendo al ahogo presupuestario, al recorte de salarios, a la competencia de las universidades privadas en el marco del discurso de la ineficiencia con que el neoliberalismo intentó deslegitimar a todas las instituciones públicas.

La Universidad de la primera década del siglo XXI se resignificó en el marco de la Ley de Financiamiento Educativo del 2006, recuperando la noción de derecho a la educación, la del Estado como garante de ese derecho en todos los niveles del sistema, haciendo efectivas esas garantías a través de múltiples programas de inclusión y de equidad; fue una universidad en la que se jerarquizó la ciencia y la tecnología, se reconocieron los derechos de la diversidad de géneros y disidencias, se amplió la vinculación con el territorio y las culturas populares.

\section{La Universidad de la primera década del siglo XXI se resignificó en el marco de la Ley de Financiamiento Educativo del 2006, recuperando la noción de derecho a la educación, la del Estado como garante de ese derecho en todos los niveles del sistema, haciendo efectivas esas garantías a través de múltiples pro- gramas de inclusión y de equidad}

Hoy, llegando al final de la segunda década del siglo XXI, nos encontramos con una ola reaccionaria a nivel mundial provocada por el "capital financiero y criminal" que proponen sociedades y ciudadanos/as despreocupados/as e insensibles por "el otro", convencidos/as que los logros son individuales, que son logros a pesar del "otro". Esta criminalidad, requiere -siguiendo a Boaventura de Sousa Santos (2019; 3)- de un "complejo ideológico-mental propagado por todo el mundo, incluyendo nuestros barrios, nuestras casas y nuestra intimidad. Son tres las fábricas principales de este complejo: la fábrica del odio, la fábrica del miedo y la fábrica de la mentira... La proliferación de estas tres fábricas es el motor de la ola reaccionaria que vivimos".

Entonces, hoy, nosotros/as como universitarios/as, desde un posicionamiento crítico y desde la idea de la ES como derecho, tenemos la tarea de desnaturalizar en cada pensamiento, en cada acción, en cada clase, en cada búsqueda teórica, en cada debate e intercambio, esas fábricas del odio, del miedo y de la mentira.

Para ello en primer lugar, es necesario pararnos en un capital fortaleza y en historias de luchas, que yo rescato como rasgos identitarios de la Universidad Pública Argentina: el cogobierno democrático, el acceso masivo, el involucramiento activo en la construcción de un proyecto político de sociedad justa y libre, la defensa irrestricta de los derechos humanos, la resistencia sostenida a los embates de proyectos neoliberales, la capacidad crítica de resignificar conceptos que en lugar de ser usados para someter al otro/a, sean concebidos para re- 
conocernos con otros/as en relaciones y proyectos emancipatorios.

Enfrentemos las fábricas del odio, del miedo y la mentira desde estos mojones de luchas colectivas guiadas por la búsqueda por la igualdad. Entendiendo a la igualdad como construcción de la política, según Diego Tatián (2019). Entendiendo a la igualdad, en la era de los DDHH y la Justicia, como redistribución y reconocimiento, según Nancy Fraser (2008).

Resistamos a la idea del mérito entendido desde el esfuerzo individual que me distingue del "otro" y que, desde esa diferencia, me hace merecedor/a. Sostengamos el sentido del esfuerzo individual como parte, como "igual" de un colectivo que se compromete, se corresponsabiliza, y se autoreconoce en todos/as los/as otros/as que forman parte necesaria e ineludible del propio logro. Otros y otras que no son sólo el cercano -familiar, amigo/a, compañero/a, del profe querido, sino también el otro/a "desconocido/a". De esos/as que dice Galeano: "se puede encontrar coterráneos en cualquier lugar del mundo y contemporáneos en cualquier lugar de la historia". Llegamos cada uno/a de nosotros/as con nuestro trabajo y nuestro esfuerzo a este logro, pero ineludiblemente siendo parte de esta "otredad". Recuperemos significados ancestrales que nos pertenecen, como el que en un saludo diario de los mayas: INLAK'ECH , que significa "yo soy otro tú" y la respuesta HALA KEN, que significa "tu eres otro yo". Y porqué no, también, desde una consigna que considero es una bandera para enfrentar estas fábricas: "La patria es el otro/a/e"

\section{Resistamos a la idea del mérito entendido desde el esfuerzo individual que me distingue del "otro" y que, desde esa di- ferencia, me hace merecedor/a. Sostengamos el sentido del esfuerzo individual como parte, como "igual" de un colecti- vo que se compromete, se corresponsabiliza, y se autoreco- noce en todos/as los/as otros/as que forman parte necesaria e ineludible del propio logro.}

En este contexto entonces, situados en este desafío. ¿Cómo pensar la Docencia, la Investigación y la Extensión desde la Educación Superior como derecho?

1-Por un lado, es necesario reafirmar la pasión por el objeto de conocimiento de cada una de las disciplinas a las cuales nos dedicamos y desde las cuales trabajamos.

Asumirnos apasionados por el objeto de conocimiento de nuestras disciplinas científicas implica asumirnos interesados por debatir, confrontar y cuestionarnos; pero cuidando de no quedarnos "encerrados" en pensamientos y debates progresistas, críticos y aislados.

Por eso, valorando -no neguemos ni desvaloricemos- el riquísimo capital que disponemos, no dejemos de preguntarnos por la comunidad mayor de la cual formamos parte: preguntarnos por el capital cultural 
incorporado, que va construyéndose a la par de la resolución de los problemas cotidianos, asentándose en los saberes ancestrales, en las costumbres y en las experiencias de cada nueva generación que crea y supera prejuicios, construyendo otros a partir de cada nueva experiencia vital.

\section{Asumirnos apasionados por el objeto de conocimiento de nuestras disciplinas científicas implica asumirnos interesa- dos por debatir, confrontar y cuestionarnos; pero cuidando de no quedarnos "encerrados" en pensamientos y debates progresistas, críticos y aislados.}

Si nos paramos desde esta perspectiva, no se trata sólo -nuevamente retomo a Boaventura de Sousa Santos (2006)- de pensar nuevas alternativas sobre los problemas siguiendo con las mismas lógicas de pensamiento; sino que el desafío es encontrar formas alternativas de pensar alternativas; o sea revisar las perspectivas y paradigmas desde los cuáles se ha venido construyendo el conocimiento científico ya que no ha servido para resolver las grandes promesas de la modernidad. El conocimiento moderno acompaña el proceso de la emergencia y consolidación de la sociedad liberal del siglo XVII con sus ideales de igualdad que no incluía a las mujeres, los indígenas, los niños, los negros. También acompaña el proceso de constitución de la sociedad capitalista y sus reglas de mercado, marcando las posiciones sociales y las reglas del poder. Este proceso histórico permite entender la hegemonía del conocimiento como regulación que no permitió resolver sino que profundizó la exclusión, la discriminación, la explotación, la insustentabilidad.

El diálogo de saberes y la traducción intercultural, entonces, no es sólo una definición que le viene bien a la extensión, es una definición que está en la base del conocimiento universitario para que avancemos desde el conocimiento-regulación al conocimiento-emancipación.

2-Otro aspecto central es preguntarnos cómo nos relacionamos -en tanto portadores de conocimiento científico- con los otros saberes existentes: de resolución de problemas cotidianos, de visiones sobre el mundo de la vida, sobre futuros posibles...

Podemos señalar por lo menos tres problemas de esa relación y consecuentemente, tres desafíos para superarlos:

- Romper el encapsulamiento -o concepción de torre de cristal; requiere reconocer al campo intelectual y académico como un campo más, donde hay disputa, intereses, construcción de límites, de disputas de poder. Es necesario liberarnos de esa aurea de "librepensadores" de la ilustración, que estamos más allá del bien y del mal, que lo mundano no nos toca por ser "educación superior".

- Romper la jerarquía y la subordinación entre saberes, requiere desarrollar el ejercicio de escucha en primer lugar, para luego poder hablar y 
hacerse presente con ese otro sujeto que sabe.

- Romper el dogmatismo, lo que implica reconocer que un mismo objeto de conocimiento puede ser visto desde distintos puntos de vista.

Ya está instalado en nuestros discursos el concepto de diálogo, pero falta mucho aún por modificar en nuestras prácticas. Seguimos reproduciendo movimientos unidireccionales en el que el saber del "experto" aporta a un otro "carente" de saber -aún bajo apariencias de reconocimiento del otro que suelen asumir perfiles paternalistas; o prácticas utilitarias que toma los problemas del medio como "ejercicios" que "sirven" para la formación profesional o que resultan "interesantes" para ser investigados. Interrogarnos permanentemente por esta "relación" es la tarea; tarea inconclusa porque necesariamente la relación crítica con la diferencia nos ubica/rá siempre en un lugar de tensión. Esa es la tarea interrogarnos permanentemente por la diferencia, que como un inconsciente colectivo pugna por aparecer. Tener dudas a la par de afirmaciones y certezas contingentes.

\section{Ya está instalado en nuestros discursos el concepto de diá- logo, pero falta mucho aún por modificar en nuestras prác- ticas. Seguimos reproduciendo movimientos unidirecciona- les en el que el saber del "experto" aporta a un otro "carente" de saber -aún bajo apariencias de reconocimiento del otro}

Esa enunciación de problemas y desafíos de superación se dice fácil, pero implica un cuestionamiento epistemológico respecto a las respuestas que la ciencia y las distintas disciplinas han estado construyendo sobre esa relación. Frente a esto de Sousa Santos propone el posmodernismo de oposición que implica tomar "la crítica de la modernidad como punto de partida para la construcción de alternativas epistemológicas y políticas" (2005:112).

3-El último aspecto central es nuestra capacidad de lectura y respuesta a la demanda social. En este punto me interesa detenerme un poco más, tal vez por mi construcción desde el Trabajo Social y desde los aportes de la función de Extensión para pensar la Educación Universitaria.

Entiendo a la demanda social "como la expresión de una agenda, de temas y de perspectivas de tratamiento de los mismos en función de la correlación de fuerzas de sectores en un momento dado; y no como la demanda unilateral de un actor o como demanda de mercado. La construcción de esta agenda de la demanda social se realiza, necesariamente, en un proceso conformado de encuentro, escucha, debate y concertación con actores sociales, económicos y políticos del medio local del cual la universidad forma parte"1. Al adoptar esta definición estamos reconociendo que nos movemos en procesos conflictivos, de lucha y disputa de sentidos y en los cuales la Universidad Pública es un actor

1 Documento "Pronunciamiento sobre la función de extensión en la Universidad pública". II Foro de Extensión Universitaria - UNC - 2 y 3 de Octubre 2008 (http://www.extension.unc.edu.ar/2o-foro-de-extension) 
político con un alto grado de poder, basado especialmente en su capital cultural y simbólico.

Entonces, es necesario desarrollar una capacidad de escucha, de análisis, de interpretación de las necesidades, porque no son unívocas las lecturas sobre las mismas, sino fruto de la constante disputa de los sentidos, donde los expertos tenemos un poder central, el poder del conocimiento. Y reconocer que participar en la resolución de la demanda social es participar de la lucha por las necesidades no es una lucha por los recursos, solamente, antes de esa lucha está la de instalar una necesidad como tal en la agenda pública, y luego la lucha por definir esa necesidad. (Fraser, 1994).

¿Desde qué lugar, como universitarios/a, ejercemos esa capacidad de lectura y construcción de la demanda social?

\section{¿Desde qué lugar, como universitarios/a, ejercemos esa capacidad de lectura y construcción de la demanda social?}

3.a- La autonomía relativa en el espacio público: la presencia de la Universidad en el medio implica ocupar una posición en un campo ${ }^{2}$ y relacionarse con otros actores de la esfera público estatal, público societal y privada. En este espacio multiactoral, la participación de las Universidades Nacionales ha pretendido estar orientada por la autonomía, que entendemos como principio intelectual y como criterio político, ya que, en un campo de relaciones donde cada actor hace prevalecer sus intereses particulares, la universidad pública -en tanto ente estatal autónomo y semiautárquico- tiene su fortaleza política en la esencia deliberativa y argumentativa que significa la toma de decisiones en el marco del cogobierno, el que nos exige tratar los temas públicos desde la consideración de los puntos de vista de los distintos claustros y además desde distintos proyectos y perspectivas políticas que en cada claustro se expresan. La autonomía universitaria no es un principio abstracto, sino que se disputa y se gana permanentemente en cada encuentro con otros actores sociales y políticos. Mientras se pueda dar cuenta de ella y se tomen posiciones ante problemas concretos, se den a conocer a la opinión pública, se expresen en los medios de comunicación, se estará alimentando la legitimidad de la universidad ante la ciudadanía.

3.b- El diálogo de saberes entre disciplinas y profesiones abre posibilidades enormes para desarrollar una capacidad de lectura de la demanda social. Debemos avanzar en las perspectivas asentadas en la integralidad, la multidimensionalidad y el pensamiento complejo en los enfoques sobre las problemáticas/temáticas/acciones a abordar, ya sea en la docencia, en la producción de conocimientos, en la intervención educativa/ comunicativa de nuestras acciones en y con actores extrauniversitarios. Ello lleva necesariamente a poner en cuestión, repensar, debatir y actualizar permanentemente los aportes dis-

2 Desde la perspectiva de Bourdieu. 
ciplinares e interdisciplinares. Si la demanda social es leída desde estos principios, necesariamente se verán interpelados los marcos teóricos disciplinares y se generará una tensión potenciadora de la interdisciplina, verificándose aquí el importante aporte de la extensión hacia adentro de la Universidad. Diálogo, Interdisciplina y Articulación, desafíos aún pendientes al interior de nuestras instituciones, ya que dentro del campo académico se continúan verificando relaciones de subordinación entre el saber científico y el saber popular, entre las ciencias físicas y naturales y las sociales/ humanas ${ }^{3}$, entre las funciones de docencia, investigación y extensión.

3.c- Reconocernos como institución social implica aceptar que las definiciones institucionales se construyen en contextos históricos y por lo tanto, entender que la relación Universidad -Estado y Universidad- Sociedad estará marcada por los modelos de Estado y de desarrollo y las concepciones teóricas e ideológicas predominantes en cada contextos histórico. Es saludable, interesante, y esclarecedor mirar nuestras trayectorias históricas como Universidades Nacionales y encontrar las tensiones, las disputas y los significados que hemos sido capaces de construir. Reconstruir las historias nos ayuda a vernos más mundanos y parte de las luchas sociales. Dejarnos interpelar por los ejes políticos del proyecto societal que se está debatiendo en el contexto histórico que nos toque vivir: hoy, el eje de la ciudadanía y los derechos humanos tiene una gran potencialidad ya que: 1) realzan el papel central e ineludible del Estado y sus políticas públicas como garantes del acceso efectivo a los derechos y al status de ciudadano/a $y, 2$ ) realzan el papel de los movimientos sociales en la construcción y renovación de agendas públicas, en la exigibilidad de los derechos humanos en general y de los derechos económicos, sociales y culturales en particular.

Para cerrar, vuelvo al profesor Parisí (2005) quien nos ha enseñado que ninguna sociedad (o mundo vincular) logra la reconciliación total consigo misma, pero sí puede trabajar y trabaja en forma permanente para disminuir su grado de conflictualidad ya que la construcción de los mundos vinculares críticos, los acuerdos, articulaciones y consensos nunca son un dato a priori sino, siempre, es el producto de una praxis colectiva, crítica e histórica; en síntesis, tienden a ser resultados y no punto de partida.

${ }^{3}$ Cuestiones centrales a discutir en el mundo académico y que generan polémicas importantes. Al respecto ver: Borón Atilio; "No somos teólogos". (columna escrita en respuesta a entrevista al Ministro Lino Barañao) Página 12 12 /01/08 y el interesante artículo de Roberto Follari "La ofensiva de la tecnociencia" en la Revista ConCiencia Social de la Escuela de Trabajo Social de la UNC, Año VI № 9 de septiembre del 2006. Pags. 105 a 112. 


\section{BIBLIOGRAFIA}

de Sousa Santos, Boaventura (2006). "Renovar la teoría crítica y reinventar la emancipación social", Bs. As.: CLACSO.

de Sousa Santos, Boaventura (2019). "Las incesantes fábricas del odio, del miedo y la mentira". Febrero 2019.

Fraser, Nancy (1994). "La lucha por la interpretación de las necesidades: esbozo de una teoría crítica socialista-feminista de la cultura política del capitalismo tardío", en Revista Debate Feminista, № 3, México, 1994.

Fraser, Nancy (2008). "La justicia social en la era de la política de identidad: redistribución, reconocimiento y participación", Revista de Trabajo. Año 4 Número 6. Agosto-Diciembre 2008

Parisí, Alberto (2005). "Diferencia" y "Contradicción/Conflicto"; en Salas Astrain, Ricardo;"Pensamiento Crítico Latinoamericano; Ediciones Universidad Silva Henríquez, Santiago, 2005.

Tatián, Diego (2018). "La igualdad como "construcción de la política”, columna de Opinión, Página 12, 26 de noviembre de 2018.

Documento "Pronunciamiento sobre la función de extensión en la Universidad pública". II Foro de Extensión Universitaria -UNC- 2 y 3 de Octubre 2008 (http://www.extension.unc.edu.ar/2o-foro-de-extension) 